\title{
O DISCURSO DO SUJEITO COLETIVO NAS EXPECTATIVAS DE MULHERES COM LOMBALGIA PARTICIPANTES DA ESCOLA DE POSTURA
}

DOI: $10.22289 / 2446-922 X . V 5 N 2 A 8$

\author{
Juerila Moreira Barreto ${ }^{1}$ \\ Maria Claudia Gatto Cardia \\ Nilton Formiga
}

\section{RESUMO}

A Escola de Postura é um método terapêutico-pedagógico das algias da coluna vertebral. Neste estudo pretende-se compreender a partir de depoimentos escrito expressos pelas participantes, as expectativas das motivações que conduziram algumas mulheres de participar do projeto de extensão Escola de Postura do curso de Fisioterapia da Universidade Federal da Paraíba. Participaram 27 mulheres, na faixa etária de 42 a 68 anos, com diagnóstico de lombalgia crônica inespecífica. Para análise foi utilizado o método do Discurso do Sujeito Coletivo com a seguinte pergunta norteadora realizada na primeira seção: "Qual a sua expectativa a respeito do trabalho cuidando da coluna vertebral desenvolvido na Escola de Postura? Observou-se quatro ideias centrais como motivadoras da participação na atividade de Extensão Escola de Posturas - UFPB: a) consciência corporal e melhorar a postura (48\%); b) expectativa de melhorar as dores da coluna (44,4\%); c) adquirir conhecimento (37\%); d) bem-estar (11,1\%); em relação a Ancoragem, identificou-se a qualidade de vida (14,8\%). Considerando tais resultados, as expectativas detectadas no encontro com as participantes, estão alinhadas com os objetivos propostos no projeto de extensão, estes, buscavam avaliar as motivações das mulheres para frequentarem a escola de posturas.

Palavras-chave: Lombalgia; Escola de Posturas; Discurso do Sujeito Coletivo.

\section{ABSTRACT}

The "Back School" is a therapeutic-pedagogical method of the spinal column. This study intends to understand from the written statements expressed by the participants, the expectations of the motivations that led some women to participate in the project of extension Back School of the Physiotherapy course of the Federal University of Paraíba. Twenty-seven women, aged 42-68 years, diagnosed with non-specific chronic low back pain, participated. For the analysis, the

\footnotetext{
${ }^{1}$ Endereço eletrônico de contato: juerila@gmail.com

Recebido em 17/06/2019. Aprovado pelo conselho editorial para publicação em 26/11/2019.
}

Rev. Psicol Saúde e Debate. Dez., 2019:5(2):112-124. 
Collective Subject Discourse method was used with the following guiding question in the first section: "What is your expectation regarding work taking care of the spine developed in the Back School? It was observed four main ideas as motivators of the participation in the activity of Extension Back School - UFPB: a) body awareness and improve posture (48\%); b) expectation of improving spine pain (44.4\%); c) acquire knowledge (37\%); d) well-being (11.1\%); in relation to Anchorage, the quality of life was identified (14.8\%). Considering these results, the expectations detected in the meeting with the participants are in line with the objectives proposed in the extension project, these sought to evaluate the motivations of women to attend the Back School

Keywords: Low back pain; Back School; Discourse of the Collective Subject.

\section{INTRODUÇÃO}

A lombalgia foi apontada como a principal causa de anos vividos com incapacidade em oitenta e seis países e a segunda ou terceira causa principal em outras sessenta e sete nações (Global Burden of Disease Study [GBDS], 2015). Embora a análise do GBDS realizado em 2010, tenha trazido mais atenção a esses transtornos, há pouca discussão de políticas de saúde sobre as opções disponíveis para abordar e prevenir os agravos da coluna vertebral (GBDS, 2015, Global Health Metrics, 2017).

As dores na coluna têm-se mostrado a causa mais prevalente dentre as alterações musculoesqueléticas, representando $78 \%$ do total das disfunções entre os usuários encaminhados para tratamento no "Grupo da Coluna", este, tem como objetivo a redução de danos gerados por dores crônicas ao atendimento a portadores de lombalgias, no qual, 38\% desses pacientes possuem queixas de dores moderadas, cujos os valores variam entre 3-6 pontos, na Escala Numérica de Dor-END) e 40\% dores intensas apresentam suas dores variando entre 6-9 pontos na END (Vieira, Nonnenmacher, Bartz, Bueno, \& Macedo, 2014).

No que se refere a dor lombar, pode ser definida como um desconforto ou dor localizada abaixo da margem costal e acima das pregas glúteas inferiores, com ou sem irradiação para os membros inferiores; sendo a dor lombar crônica caracterizada por persistir por pelo menos 12 semanas (Airaksinen, et al. 2005). A dor lombar é classificada de acordo com a duração dos sintomas sendo: Aguda, com duração inferior a 6 semanas; subaguda, entre 6 e 12 semanas e a crônica, com duração superior a 12 semanas (Airaksinen et al. 2005; Chou et al. 2007). Essas disfunções têm sido tratadas também em formato grupo como o modelo da Escola de Postura.

A Escola de Posturas (EP), originalmente, denominada "Back School", é também conhecida no Brasil como Escola de Coluna; trata-se de um método que serve para o orientação postural que previne e trata pacientes com dor lombar, compõe-se de informações teóricoeducativa com práticas de exercícios terapêuticos para a coluna; porém, com a aplicação deste método, surgiram algumas modificações na forma de aplicação e nos parâmetros de avaliação

Rev. Psicol Saúde e Debate. Dez., 2019:5(2):112-124. 
em diferentes locais onde a escola é adotada, para melhor adaptação de cada realidade, porém sem fugir do seu fundamento principal que é a compreensão da relação da dor com o aumento de tensão mecânica na realização das atividades da vida diária (Cardia, 2018, Noll, Vieira, Darski, \& Candotti 2013).

A EP foi desenvolvida na Suécia em 1969, no Hospital Danderyd sob a direção de Zachrisson-Forssel, com objetivo de gerar uma prática de autoajuda para os trabalhadores em geral, a qual, destinou-se ao cuidado com as costas (back care) a partir de aconselhamentos ergonômicos. O modelo precursor sueco reforça a importância das aulas educativas utilizando material didático exigindo resultados na mudança dos hábitos posturais. Estas são características que favorecem a promoção da saúde (Cardia, 2018).

O método da EP faz parte das atividades de extensão do curso de Fisioterapia da Universidade Federal da Paraíba, na cidade de João Pessoa há 29 anos, atendendo homens e mulheres, da adolescência a terceira idade. Teve início a partir de 1990, como projeto de extensão cujo objetivo era atender pessoas com alterações posturais e dores na coluna, a partir de orientações teórico-práticas, exercícios terapêuticos e relaxamento, tendo em vista contribuir na orientação postural. (Cardia, 2006; Cardia, 2018).

Segundo Andrade, Araújo, Ribeiro, \& Vilar, 2005), a "Escola de Coluna", inclui os seguintes aspectos: a) noções de anatomia e fisiologia básica da coluna; b) epidemiologia e fatores causadores da dor lombar; $c$ ) informações sobre como reduzir a intensidade e frequência da dor lombar com a modificação da postura nas atividades de vida diária; d) o valor dos exercícios para manutenção de na coluna saudável.

A EP da UFPB possibilita informações diversas sobre ergonomia, controle do sistema neuroendócrino, da dor e da respiração e abrangem também, uma prática de exercícios terapêuticos com abordagens muito variadas (Cardia et al., 2006). Com este conceito expandido, a EP/UFPB visa a conscientização corporal para mudanças de hábitos posturais e controle da dor com programas bastantes flexíveis.

As Escolas de Posturas em geral possuem perfis bastantes heterogêneos, com variedades de números de aulas, publico alvo, metodologia das aulas, profissionais envolvidos; porém, tanto a nível nacional, quanto internacional, os resultados tendem a ser positivos no tratamento das afecções na coluna (Santos \& Moreira, 2009).

Um estudo de revisão sistemática da biblioteca Cochrane, realizado por Heymans, Van Tulder, Esmail, Bombardier e Koes (2008), no qual foi analisado um total de 19 programas de Escola de Posturas (3584 pacientes), e concluíram que apenas 6 ensaios possuíam alta qualidade metodológica. Os resultados demonstraram evidência moderada de que as EP proporcionam melhores efeitos sobre a dor e o estado funcional recorrente e crônico. Também 
verificaram evidência moderada para o controle da dor lombar crônica em âmbitos ocupacionais e de promoção da saúde.

A EP enquanto um método educativo-terapêutico se alinha com a educação em saúde permitindo que as pessoas participantes das atividades possam interagir de uma forma mais ampla com o seu processo pessoal na promoção, manutenção e restauração da saúde de forma coletiva (em grupo). Conhecer a narrativa das participantes de um programa de educação em saúde permite ao terapeuta/gestor maior clareza no direcionamento da aplicação de saberes, direcionados ao desenvolvimento humano; nessa perspectiva conhecer o discurso de uma coletividade fortalece as ações realizadas em busca do bem-estar e melhoria da qualidade de vida (Araújo et al. 2013).

O discurso do sujeito coletivo (DSC) é uma forma de recuperar e expressar as representações sociais adquiridas da experiência vivida (empíricas), em que as opiniões ou expressões individuais que apresentam sentidos semelhantes são agrupadas em categorias semânticas gerais, como normalmente se faz quando se trata de perguntas ou questões abertas (Lefèvre, 2017).

O DSC é uma técnica de processamento de dados qualitativos, principalmente de depoimentos, com vistas à obtenção e descrição dos sentidos das opiniões ou representações coletivas. A obtenção do discurso está subdividida em várias etapas, efetuado por meio de quatro operadores elaborados sobre o material verbal ou escrito coletado nas pesquisas. São eles: Expressões Chave (ECH), Ideia Central (IC), Ancoragens (AC) e o Discurso do Sujeito coletivo (DSC) propriamente dito. (Lefèvre \& Lefèvre, 2006)

As expressões chave $(\mathrm{ECH})$ são pedaços, trechos do discurso, que devem ser destacados pelo pesquisador, e que revelam a essência do conteúdo do discurso ou a teoria subjacente. A ideia central (IC) é um nome ou expressão linguística que revela, descreve e nomeia, da maneira mais sintética e precisa possível, o(s) sentido (s) presentes em cada uma das respostas analisadas e de cada conjunto homogêneo de ECHs, que vai dar origem, posteriormente, ao DSC. A ancoragem (AC) é a expressão de uma dada teoria ou ideologia que o autor do discurso professa e que está embutida no seu discurso como se fosse uma afirmação qualquer. O discurso do sujeito coletivo (DSC) é uma reunião em um só discurso-síntese redigido na primeira pessoa do singular de $\mathrm{ECH}$ que têm IC ou AC semelhantes ou complementares (Lefèvre, Lefèvre, Cardoso, \& Mazza, 2002; Figueiredo, Chiari, \& Goulart, 2013)

A partir das concepções destacadas acima este trabalho tem como objetivo compreender as falas expressas no levantamento das expectativas das motivações que conduziram algumas mulheres de participar do projeto de extensão Escola de Postura, no curso de fisioterapia em uma instituição de ensino superior pública, no estado da Paraíba na cidade de João Pessoa. 


\section{MATERIAS E MÉTODOS}

\section{Participantes}

Participaram do projeto de extensão Escola de Postura, 27 mulheres, com idades de 42 a 68 anos, com diagnóstico de lombalgia inespecífica, no período de janeiro 2017 a março 2018, oferecido pelo curso de Fisioterapia numa instituição de ensino superior no estado da Paraíba.

\section{Instrumento de coleta de dados}

O instrumento constou de duas partes, descritas a seguir:

a) Questionário sócio demográfico: neste, encontravam-se questões referentes a idade, sexo, IMC, estado civil, etnia, religião, renda econômica, nível educacional e avaliação da coluna

b) Uma pergunta aberta no momento de levantamento da expectativa quanto a participar do projeto, que foi a seguinte:

"Qual a sua expectativa a respeito do trabalho cuidando da coluna vertebral desenvolvido na Escola de Postura?

\section{Procedimento para coleta de dados}

A coleta de dados ocorreu durante a realização de um grupo especifico para mulheres pertencentes ao projeto de Extensão: Escola de Posturas da UFPB, realizado no departamento de Fisioterapia da UFPB, que apresentou a seguinte estrutura metodológica:
a) Tempo de realização: 16 sessões,
b) Tempo de execução da atividade: 180 minutos;
c) Periodicidade: uma vez por semana;
d) Etapas do trabalho estão distribuídas em três momento: 1) Momento afetivo no qual eram utilizadas técnicas de dinâmica de grupo com o objetivo de inclusão e desenvolvimento afetivo; 2) Momento cognitivo que se destinava a apresentação de informações teóricas referentes a coluna vertebral e suas disfunções; 3) Momento de atividade motora, no qual eram desenvolvidas atividades práticas como: a) Auto reconhecimento corporal, b) massagem na plantas dos pés com bastão; c) automassagem com a colher; d) Exercícios respiratórios; e) Estabilização segmentar lombar; f) Atividade aeróbica de baixo impacto como Dança Circular.

Para o presente artigo, foi utilizado informações referentes a $1^{\text {a }}$ sessão, na qual, foi realizado o levantamento das expectativas a respeito da atividade a seria desenvolvida ao longo das 16 sessões. 


\section{Procedimento para análise dos dados}

Para a realização dessa análise foi utilizada a técnica do DSC que é uma proposta de organização e tabulação de dados qualitativos de natureza verbal ou escrito, obtidos de depoimentos. (Lefèvre, 2017); além dessa, realizou-se o calculo de percentagem, com objetivo de verificar a frequência, em percentual, da quantidade de expressões apresentadas pelas participantes.

\section{Considerações éticas}

As participantes do estudo foram informadas tanto por escrito quanto verbalmente das normas que disciplinavam a atividade e o seu vínculo com a pesquisa, e aquelas que aceitaram participar assinaram o Termo de Consentimento Livre e Esclarecido (TCLE) conforme estabelece a Resolução 466/2012. A pesquisa foi aprovada pelo Comitê de Ética em Pesquisa do Centro de Ciências da Saúde da Universidade Federal da Paraíba - CEP/CCS sob o número CAAE: 31639413.7.0000.5188>>

\section{RELATO DA EXPERIENCIA}

Após análise das repostas por escrito da pergunta norteadora realizada na $1^{\text {a }}$ sessão (Qual a sua expectativa a respeito do trabalho cuidando da coluna vertebral desenvolvido na Escola de Postura?); destacamos quatro ideias centrais como motivadoras da participação na atividade de Extensão Escola de Postura: a) consciência corporal e melhorar a postura (48\%); b) expectativa de melhorar as dores da coluna (44,4\%); c) adquirir conhecimento (37\%); d) bemestar (11,1\%). E como Ancoragem identificamos a qualidade de vida 1(4,8\%).

No Quadro 1, são apresentadas as Expressões chaves $(E C H)$; na sequência foi identificada a ideia central (IC) e elaborado o discurso do sujeito coletivo (DSC); abaixo apresentado. Como a ideia mais recorrente foi a busca por uma melhor consciência corporal e melhorar a postura com 48\%, sendo uma motivação importante para participar do grupo da Escola de Postura.

Quadro 1: Expressões Chaves (ECH) e ideia central (IC) de 13 sujeitos

\begin{tabular}{|l|l|l|}
\hline Sujeito & $\begin{array}{l}\text { Expressão Chaves } \\
(\mathrm{ECH})\end{array}$ & $\begin{array}{l}\text { Ideia central } \\
\text { IC }\end{array}$ \\
\hline 1 & $\begin{array}{l}\text { Fazer atividades do dia-a-dia que não prejudicar a } \\
\text { coluna. }\end{array}$ & \\
\hline
\end{tabular}

Rev. Psicol Saúde e Debate. Dez., 2019:5(2):112-124. 


\begin{tabular}{|l|l|l|}
\hline 2 & Melhorar minha postura & \\
3 & Melhorar minha postura/coluna & $\begin{array}{l}\text { Consciência } \\
\text { corporal e melhorar } \\
\text { a postura }\end{array}$ \\
4 & Espero melhorar a coluna/postura & \multirow{2}{*}{ Tro } \\
18 & Andar mais alinhada & \\
19 & Prestar atenção no que está acontecendo comigo & \\
20 & Ter uma postura melhor & \\
22 & Manter a postura & \\
24 & Melhorar a minha postura & \\
25 & Melhorar a minha postura & \\
27 & Ter uma postura adequada para a coluna & Minha postura em ordem
\end{tabular}

DSC referente ao quadro 1 é destacado a seguir:

Eu preciso prestar mais atenção no que está acontecendo comigo. Espero melhorar minha postura/coluna. Ter e manter uma postura melhor e em ordem, está, mas alinhada. Ter uma postura adequada para a coluna. Com trabalho corporal fazer atividades do dia-a-dia que não prejudique a coluna.

Em estudos realizados por Caromano, et al. 2011, utilizando técnicas de auto avaliação e autocuidado corporal (TAAC) com objetivo de ganho do conhecimento corporal e diminuição da dor e desconforto musculo esquelético obteve ganhos significativos na utilização de várias técnicas que propiciam uma maior percepção corporal tais como percussão óssea, escovação da pele, automassagem e alongamento. No Quadro 2, tem-se como a $2^{\underline{a}}$ ideia mais recorrente com $44,4 \%$, expectativa de melhorar as dores da coluna.

Quadro 2: Expressões Chaves (ECH) e ideia central (IC) de 12 sujeitos

\begin{tabular}{|l|l|l|}
\hline Sujeito & $\begin{array}{l}\text { Expressão Chaves } \\
\text { (ECH) }\end{array}$ & $\begin{array}{l}\text { Ideia central } \\
\text { (IC) }\end{array}$ \\
\hline 5 & Melhorar minha coluna das dores & \\
6 & Melhorar as dores da coluna & \\
9 & Ficar boa das dores. Cuidar da coluna & \\
11 & Melhorar as minhas dores. Conseguir uma vida melhor & \\
12 & Espero melhorar as condições da postura & Expectativa de \\
\cline { 2 - 3 }
\end{tabular}

Rev. Psicol Saúde e Debate. Dez., 2019:5(2):112-124. 


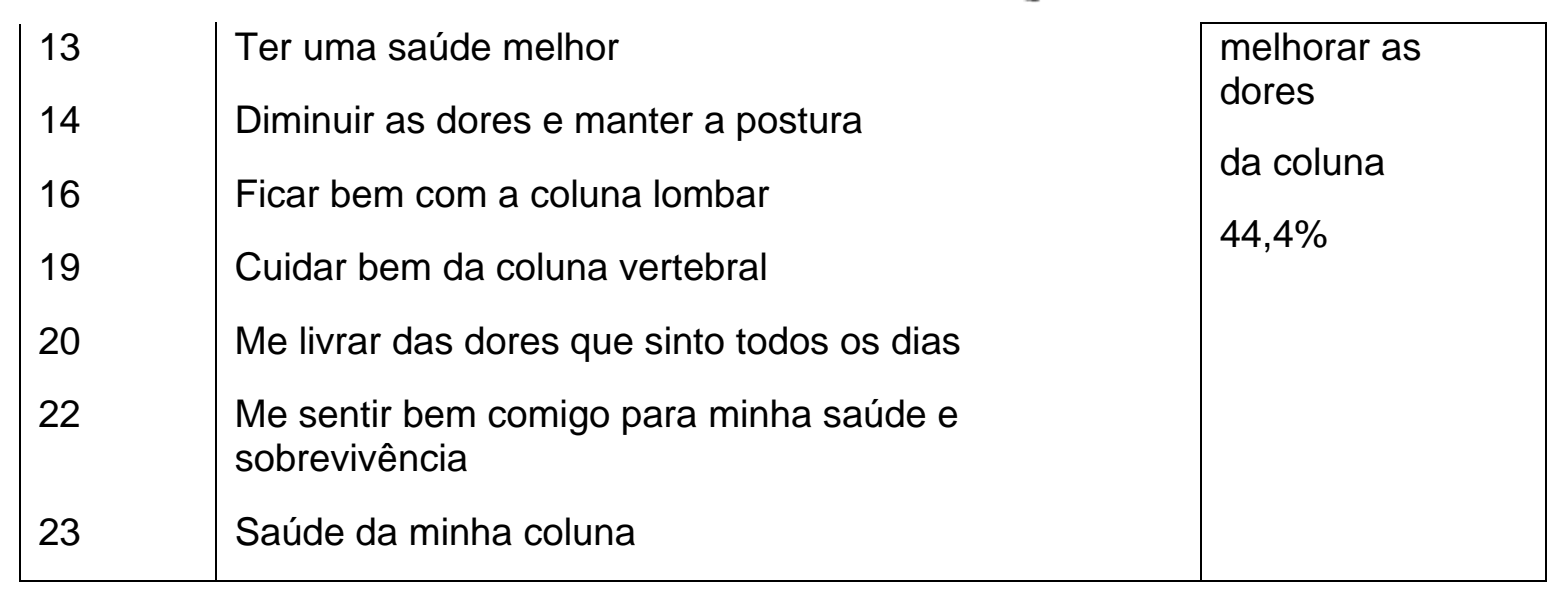

\section{DSC}

Espero melhorar e ficar boa das minhas dores da coluna. Me livrar dessas das dores que sinto todos os dias e melhorar as condições da postura. Cuidar bem da coluna vertebral/lombar e ter uma saúde melhor. Me sentir bem comigo para minha saúde da minha coluna e sobrevivência. Conseguir uma vida melhor.

Essa expectativa de melhora nos conduz ao construto "otimismo" destacado por Santos \& Wechsler, (2015), que se refere a expectativas generalizadas favoráveis com relação a acontecimentos futuros e, de modo geral, influencia a maneira como a pessoa percebe a si mesma e o mundo ao seu redor. Níveis mais altos de otimismo foram relacionados a melhor bem-estar subjetivo em tempos de adversidade ou dificuldade. Há evidências de que o otimismo está associado à ações proativa para proteger a saúde, estando o otimismo relacionado aos indicadores de melhor saúde física. (Carver, Scheier, \& Segerstrom, 2010).

No Quadro 3, como terceira ideia central, adquiri conhecimento, destaca-se que 37\% das participantes quer dá sentido ao conteúdo expresso nas expressões chave.

Quadro 3: Expressões Chaves (ECH) e ideia central (IC) de 10 sujeitos

\begin{tabular}{|c|c|c|}
\hline Sujeito & $\begin{array}{l}\text { Expressão Chaves } \\
\text { (ECH) }\end{array}$ & Ideia central (IC) \\
\hline 1 & $\begin{array}{l}\text { Aprender a melhorar a postura, a deitar, levantar, sentar, } \\
\text { andar. }\end{array}$ & \\
\hline 3 & Adquiri conhecimento. & \\
\hline 10 & Aprender a postura correta & \\
\hline 12 & $\begin{array}{l}\text { Aprender métodos terapêuticos ou físicos para suportar os } \\
\text { sintomas }\end{array}$ & $\begin{array}{l}\text { Adquirir } \\
\text { conhecimento }\end{array}$ \\
\hline 13 & $\begin{array}{l}\text { Aprender estratégias para uma postura mais adequada. } \\
\text { Partilhar experiências de vida }\end{array}$ & \\
\hline
\end{tabular}

Rev. Psicol Saúde e Debate. Dez., 2019:5(2):112-124. 


\begin{tabular}{|l|l|}
14 & Mais conhecimento \\
15 & Aprender novas habilidades e correções da postura \\
17 & Conhecimento teórico e prática física \\
20 & Conhecimento do meu corpo
\end{tabular}

\section{DSC}

Para eu adquiri mais conhecimento sobre meu corpo, através de teoria e prática física, aprender novas habilidades e correções da postura, adquirir estratégias para uma postura mais adequada para melhorar e ter a postura correta ao deitar, levantar, sentar, andar. Aprender métodos terapêuticos ou físicos para suportar os sintomas. Aprender a entender os meus problemas de saúde e partilhar as minhas experiências de vida.

A aprendizagem é um elemento intrínseco da condição humana. Aprendemos a todo o momento, em um processo de interação permanente com o meio, manifestando diferentes níveis de complexidade referentes ao conhecimento construído (Bridi \& Bridi, 2019). A aprendizagem pode ser definida como um processo de aquisição de novos conhecimentos através de experiências vivenciadas e determinadas por fatores endógenos e exógenos que resultam na modificação do comportamento humano e que dependem de condições essenciais tais como: mentais, física, sensoriais e sociais (Neto \& Costa, 2017)

Aprender a reconhecer a linguagem do corpo, torna-se um ponto crucial na reorganização das atividades da vida diária (AVDs). Ao detectar possível alterações que produzam sobrecarga e dor, através de uma maior percepção sensorial começando pela pele que é inervada por fibras nervosas que se originam de um único gânglio nervoso dorsal (dermátomos), depois os músculos supridos por um segmento espinhal e seu par de nervo espinhal (miotómos); permite maior expansão da consciência favorecendo a movimento corporais cuidadosos e auto protetores na relação com o ambiente (Natour, 2004).

No Quadro 4, como quarta ideia central, bem-estar, destacamos que 11,1\% que dá sentido ao conteúdo expresso nas expressões chave.

Quadro 4: Expressões Chaves (ECH) e ideia central (IC) de 3 sujeitos

\begin{tabular}{|l|l|l|}
\hline Sujeito & $\begin{array}{l}\text { Expressão Chaves } \\
(\mathbf{E C H})\end{array}$ & $\begin{array}{l}\text { Ideia central } \\
\text { (IC) }\end{array}$ \\
\hline 7 & $\begin{array}{l}\text { Espero coisas boas para minha saúde. A dor nos deixa de } \\
\text { mal humor e de mal com a vida } \\
\text { Espero viver melhor. Bem-estar }\end{array}$ & Bem-estar \\
\hline
\end{tabular}

Rev. Psicol Saúde e Debate. Dez., 2019:5(2):112-124. 


\begin{tabular}{|l|l|l|} 
& Melhorar a minha autoestima & $11,1 \%$ \\
\hline
\end{tabular}

\section{DSC}

A dor nos deixa de mal humor e de mal com a vida, então espero coisas boas para minha saúde. Espero viver melhor. Bem-estar. Melhorar a minha autoestima

O bem-estar relacionadas as emoções e sentimentos das pessoas (Farsen, Ribeiro, Boehs, \& Silva, 2018). O conceito de bem-estar esteve relacionado aos estudos da área econômica e relativo ao bem-estar material (Welfare), a partir da década de 60 a concepção ultrapassou a dimensão de bem-estar econômico se ampliou e se constituiu para uma dimensão, mais global, passando a considerar o bem-estar como uma medida avaliativa da vida como um todo, incluindo aspectos subjetivos que favoreceu um aumento dos estudos nos anos de 1960 a 1980 (Galinha, 2008).

No Quadro 5, destacamos como Ancoragem, a Qualidade de vida, em que 14,8\% das participantes expressão esse construto que aparece como uma crença que toca o texto, que dá sentido ao conteúdo expresso nas expressões chave.

No Quadro 5, nos permite visualiza a Ancoragem, na expressão Qualidade de vida, onde destacamos que $14,8 \%$, apresenta uma compreeenção maior como um estilo de vida.

Quadro 5: Ancoragens (AC) 4 sujeitos

\begin{tabular}{|l|l|l|}
\hline Sujeito & $\begin{array}{l}\text { Ancoragens } \\
\text { (AC) }\end{array}$ & DSC \\
\hline 5 & Mais qualidade de vida & Qualidade de \\
6 & Melhorar minha qualidade de vida \\
10 & Melhorar minha qualidade de vida & \\
21 & Obter qualidade de vida & $14,8 \%$ \\
\hline
\end{tabular}

\section{DSC}

Obter e melhorar a minha qualidade de vida.

A compreensão sobre a qualidade de vida envolve inúmeros campos do conhecimento humano, como o biológico, social, político, econômico, médico entre outros, numa constante inter-relação. A presença do termo qualidade de vida é facilmente percebida no linguajar da sociedade contemporânea, sendo incorporado ao vocabulário popular com várias formas de conotação. O senso comum se apropriou desse objeto de forma a resumir melhorias ou um alto padrão de bem-estar na vida das pessoas, sejam elas de ordem econômica, social ou emocional 
(Almeida, 2012). Embora "qualidade de vida" esteja mais voltada a saúde física e as questões objetivas que envolvem os seres humanos (Farsen, et al 2018).

\section{CONSIDERAÇÕES}

A partir desses resultados, o qual, pretendeu não apenas avaliar o discurso das participantes diante de um fenômeno de grande impacto na saúde destes, mas, com base nas expressões emitidas por elas, oferecer visibilidade as motivações que levaram as mulheres a participar do grupo EP e suas metas para uma melhor qualidade de vida.

A etapa inicial dos trabalhos em grupo no projeto EP é muito importante permitindo ao profissional alinhar a proposta terapêutica e as necessidades apresentadas pelas participantes favorecendo ao desenvolvimento individual e coletivo das mesmas; este momento, não apenas contempla a condição de vínculo humano e afetivo, mas, também, a identificação das motivações que a levaram até o projeto, procurando com isso fazer uma levantamento de queixasjustificativas para que os profissionais do EP tenham melhor clareza sobre a intervenção a ser aplicada.

Sendo assim, na EP foi possível apreender as expectativas das participantes para um melhor encaminhamento do protocolo de assistência, capaz de permitir uma ampliação da consciência corporal, melhora da postura, das dores da coluna, bem como, aquisição de conhecimento sobre coluna vertebral das mesmas. Tal condição, não apenas visa contribuir para uma educação em saúde do corpo, mas, também, na busca do bem-estar, associadas a qualidade de vida, adequadas a realidade individual de cada participante.

Ao avaliar esses resultados, a partir do discurso do sujeito coletivo, é possível refletir na seguinte direção: as participantes reconhecem a necessidade de uma melhor consciência corporal e da postura, a qual, permitirá o reconhecimento, de forma a prevenir, o uso inadequado do corpo e das disfunções do mal uso do corpo em sua estrutura física; haja vista que frente a essa atividade da EP, espera de foram eficiente uma melhora nas dores da coluna, favorecendo, consequentemente, um Bem-estar físico e emocional, conduzindo para uma experiência de uma vida, qualitativamente, saudável.

Por fim, acredita-se que o presente tenha cumprido com os seus objetivos e seja uma contribuição tanto para área da saúde em geral (especificamente, à atenção fisioterapêutica) e afins (Psicologia, Serviço Social, Terapia Ocupacional, etc.) 


\section{REFERÊNCIAS}

Araujo, V.S., Guerra, C.S., Moraes, M.N., Silva, J.B., Monteiro, C.Q.A., \& Dias, M. D. (2013). Discourse of the Collective Subject regarding Education of Health in the Aging Process: a descriptive study. Online brazilian journal nursing, 12 (2): 565-73. Web site: http://www.objnursing.uff.br/index.php/nursing/article/view/4093

Andrade, S. C., Araújo, A. G. Ribeiro., \& Vilar, M. J. P. (2005) Escola de Coluna: Revisão histórica e sua aplicação na lombalgia crônica. Revista Brasileira de Reumatologia. 45(4), 224-8.

Airaksinen, O., Brox, J. I., Cedraschi, C., Hildebrandt, J., Klaber-Moffett, J., Kovacs, F., et al. (2004). European guidelines for the management of chronic nonspecific low back pain. November, Amended version June.

Almeida, M. A. B., (2012) Qualidade de vida: definição, conceitos e interfaces com outras áreas, de pesquisa. São Paulo: Escola de Artes, Ciências e Humanidades. EACH/USP.

Global Burden of Disease Study 2013 Collaborators. (2015) Global, regional, and national incidence, prevalence, and years lived with disability for 301 acute and chronic diseases and injuries in 188 countries, 1990-2013: a systematic analysis for the global Burden of Disease Study 2013. Lacent, 386, 743-800.

Global Health Metrics (2017) Global, regional, and national incidence, prevalence, and years lived with disability for 328 diseases and injuries for 195 countries, 1990-2016: a systematic analysis for the Global Burden of Disease Study. Lancet. 390,1211-1259.

Bridi Filho, C. A., \& Bridi, F. R.S. Sobre o aprender e suas relações: interfaces entre neurologia, psicologia e psicopedagogia. Web site: http://srvd.grupoa.com.br/uploads/imagensExtra/legado/R/ROTTA_Newra_Tellechea/Neuro logia_Aprendizagem/Lib/Amostra.pdf Acesso em 02/06/2019 21:23".

Chou R., Qaseem A., Snow V., Casey D., Cross J.T, Jr., Shekelle P., \& Douglas K. (2007) Diagnosis and treatment of low back pain: a joint clinical practice guideline from the American College of Physicians and the American Pain Society. Annals of Internal Medicine, 147(7), 478-491.

Cardia, M. C. G. (2006) Manual da escola de postura. (3ed.) João Pessoa: Editora Universitária/UFPB.

Cardia M.C.G. (2018) Escolas de Posturas da UFPB. Projeto de Extensão da Universidade Federal da Paraíba, Departamento de Fisioterapia Web site: http://www.prac.ufpb.br/prac/extra-muros/contents/5-edicao/noticias/6-noticias/escola-deposturas-da-ufpb.

Caromano, F. A., Dias, M. C. S., Moreira, M. C. S., Carlis, M., Panades, L. S. C., Exposito, A. L., \& Miranda, C. S. (2011) Efeitos da técnica de auto avaliação e autocuidado corporal (TAAC) na percepção de dor e desconforto da região de cabeça e pescoço. Cadernos de PósGraduação em Distúrbios do Desenvolvimento, 11(1), 89-98.

Carver, C. S., Scheier, M.F., \& Segerstrom, S.C. (2010). Optimism. Clinical Psychology Review 30, 879-889.

Figueiredo, M. Z. A., Chiari, B. M., \& Goulart, B. N. G. (2013) Discurso do sujeito coletiva: uma breve introdução à ferramenta de pesquisa qualiquantitativa. Distúrbios da Comunicação, 25(1), 129-136. 


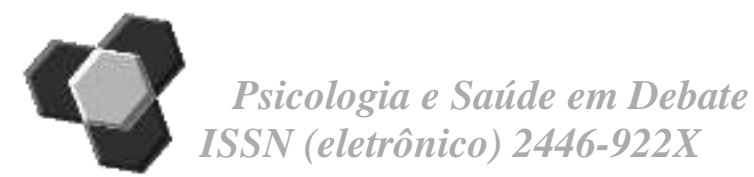

Farsen, T. C., Ribeiro, A., Boehs, S., \& Silva, N. (2018) Qualidade de vida, Bem-estar e Felicidade no Trabalho: sinônimos ou conceitos que se diferenciam? Interação em Psicologia 22(01) 31-41.

Galinha, I. (2008). Bem-estar subjectivo: Factores cognitivos, afectivos e contextuais. Coimbra: Quarteto Editora.

Heymans, M. W., Van Tulder, M. W., Esmail, R., Bombardier, C., \& Koes, B.W. (2008). Escuelas de espalda para el dolor lumbar inespecífico. (Traduzida de The Cochrane Library, Issue 2. Chichester, UK: John Wiley \& Sons, Ltd.) Web site: http://www.update-software.com.

Lefèvre, F. (2017). Discurso do sujeito coletivo. Nossos modos de pensar, nosso eu coletivo. São Paulo; Andreoli.

Lefèvre, F., \& Lefèvre, A. M. C. (2006). A proposta do DSC. Web. Site: www.ipdsc.com.br.

Lefèvre, A. M. C., Lefèvre, F., Cardoso, M. R. L., \& Mazza, M. M. P. R. (2002) Assistência pública à saúde no Brasil: estudo de seis ancoragens. Saúde e Sociedade. 11(2), 35-47

Netto, A. P., \& Costa, O.S. (2017) A importância da psicologia da aprendizagem e suas teorias para o campo do ensino-aprendizagem. Fragmentos de Cultura, 27(2), 216-224.

Natour, J et al. (2004). Coluna vertebral. São Paulo.

Noll M., Vieira A, Darski C, Candotti C. T. (2013) Escolas posturais desenvolvidas no Brasil: revisão sobre os instrumentos de avaliação, as metodologias de intervenção e seus resultados. Revista Brasileira de Reumatologia. 54(1), 51-58.

Santos, C. B. S., \& Moreira, D. (2009). Perfil das escolas de posturas implantadas no Brasil. Semina: Ciências Biológicas e da Saúde, 30 (2), 113- 120.

Santos, M. C., \& Wechsler, S. M., (2015) Análise das publicações científicas sobre otimismo em saúde no último triênio. Psicologia Argumento, 33(83), 470-482.

Vieira, A., Nonnenmacher, L. F.Q., Bartz, P.T., Bueno, A.F., \& Macedo, D.S. (2014) Perfil de usuários com dores musculoesqueléticas crônicas encaminhadas ao "grupo da coluna ". Revista Baiana Saúde Pública, 38(3), 571-584. 